\title{
Feature Diagrams as Package Dependencies ${ }^{\star}$
}

\author{
Roberto Di Cosmo and Stefano Zacchiroli \\ Université Paris Diderot, PPS, UMR 7126, Paris, France \\ roberto@dicosmo.org, zack@pps.jussieu.fr
}

\begin{abstract}
FOSS (Free and Open Source Software) distributions use dependencies and package managers to maintain huge collections of packages and their installations; recent research have led to efficient and complete configuration tools and techniques, based on state of the art solvers, that are being adopted in industry. We show how to encode a significant subset of Free Feature Diagrams as interdependent packages, enabling to reuse package tools and research results into software product lines.
\end{abstract}

Keywords: software product lines, open source, package, component, feature diagram, dependencies

\section{Introduction}

Feature models [11] are essential devices to reason about software product lines (SPLs). As features and their interdependencies get more complex, managing models quickly turns into a non-trivial task for humans. Researchers have worked to improve the situation by establishing connections between Feature Diagrams (FD) and notions like grammars [6], propositional logics [2], and constraint programming [3], paving the way to use automatic tools like SAT solvers $[9,12]$ and proof assistants [10] for SPL configuration.

Meanwhile FOSS distributions like Debian, Red Hat, and Suse have used for the past 15 years packages and dependencies to maintain some among the largest software collections known: package managers are used daily to maintain millions of installations built by selecting components from repositories of tens of thousands packages. Recent research efforts $[1,4,13,16]$ have led to the development of efficient and complete configuration and maintenance tools, as well as metrics for FOSS distributions, based on state of the art solvers, which are rapidly being adopted in industry.

We show how a significant subset of Free Feature Diagrams can be compactly encoded as interdependent packages, opening the way to massive reuse in SPLs of research results and tools coming from FOSS research. In particular, package management tools are able to scale up to tens of thousands components and hundreds of thousands dependencies, and cope well with component evolution, which is routine in FOSS. Both aspects may be of great interest for the SPL community.

* This work is partially supported by the European Community FP7, MANCOOSI project, grant agreement n. 214898. 


\section{Package dependencies for distribution maintenance}

In FOSS distributions a package is a bundle that ships a (software) component, the data needed to configure it, and metadata which describe its attributes and expectations on the deployment environment [7]. For simplicity, we focus on packages as used in the Debian distribution, but the discussion applies almost unchanged to other popular package formats like RPM (see [13] for details).

Here is a sample metadata excerpt from the firefox package:

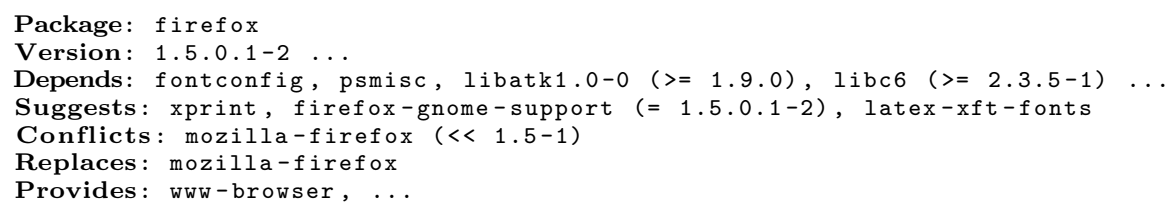

Every package has a version that is used to give a temporal order to the packaged component release. The kinds of relationships that can be expressed in the metadata of a package $\mathrm{p}$ are numerous, but the most important are:

- Depends: a list of package disjunctions $p_{1}|\ldots| p_{n}, \ldots, q_{1}|\ldots| q_{m}$, where each atom can carry a version predicate (e.g. $\geq 1.9 .0)$. For the owner package to be installable, at least one package in each disjunction must be installed.

- Conflicts: a list of package predicates $p_{1}, p_{2}, \ldots p_{n}$. For the owner package to be installable, none of the $p_{i}$ must be installed. Self conflicts are ignored.

- Recommends similar to Depends, it indicates an optional dependency; it might be advisable to satisfy it, but it is not needed to obtain a working system.

A repository $R$ is a set of packages; a subset $I \subseteq R$ of it is said to be a healthy installation if all dependencies of packages in $I$ are satisfied, and none of the conflicts is. Precise formal meanings to all these notions have been given elsewhere (see [8,13] and the Mancoosi project http://www.mancoosi.org). Tools are available in the distribution world to choose healthy installations according to user requests [15] and to perform sophisticated repository analysis $[1,5]$.

\section{Encoding feature diagrams as package dependencies}

We show how a core subset of Feature Diagrams (FD) can be compactly encoded as packages. Due to the differences among FD formalisms, we provide the encoding for a significant subset of Free Feature Diagrams (FFD) [14] that captures many known formalisms, and allows to claim that our encoding is of general interest.

FFD is a general framework that allows to capture different classes of FD by specifying a few parameters: the kind of graph $G T$ (Tree or DAG); the node type $N T$ (and, or, xor, or opt; the latter encoding explicit optionality within a node-based semantics [14]); the graphical constraint type $G C T$ ( $\Rightarrow$ for implication, and | for mutual exclusion); and the textual constraint language $T C L$ (usually including just implication and mutual exclusion, noted $n$ implies $n^{\prime}$ and $n$ mutex $n^{\prime}$, respectively). 
Definition 1 (Free Feature Diagram). $d \in F F D(G T, N T, G C T, T C L)=$ $(N, P, r, \lambda, D E, C E, \Phi)$ where:

$-N$ is a set of nodes

$-P \subseteq N$ is a set of primitive nodes

$-r \in N$ is the root node

$-\lambda: N \rightarrow N T$ labels each node with an operator from $N T$

- $D E \subseteq N \times N$ is the set of decomposition edges; $\left(n, n^{\prime}\right) \in D E$ is noted $n \rightarrow n^{\prime}$

$-C E \subseteq N \times G C T \times N$ is the set of constraint edges

$-\Phi \subseteq T C L$ are the textual constraints

A few well-formedness constraints are imposed: only $r$ has no parent; the decomposition edges do not contain cycles; if $G T$ is Tree, then $D E$ forms a tree; nodes are labeled with operators of the appropriate arity.

Precise formal semantics of FFD is given in terms of valid models [14]:

Definition 2 (Valid model). A valid model of a feature diagram $d$ is $M \subseteq$ $N$ such that: (a) $r \in M$, (b) $M$ satisfies the operators attached to each node, as well as all the (c) graphical and (d) textual constraints, with the additional requirement that (e) if a node is in the model, then at least one of its parents (called the justification) is in the model too.

We call $F F D_{\text {core }}$ the fragment of FFD obtained by restricting the operators in NT to or, and, xor, opt, and the operators in GCT and $T C L$ to implies and mutex; this fragment is enough to cover several well known Feature Diagram formalisms (OFT, OFD, RFD, VBFD, GPFT and PFT in the classification given in [14]).

We will now show how to encode any $d \in F F D_{\text {core }}$ as a package repository $R(d)$, so that valid models correspond to healthy installations of a specific package $p_{r} \in R(d)$.

Definition 3 ( $F F D_{\text {core }}$ encoding). Let $d \in F F D_{\text {core }}(G T, N T, G C T, T C L)=$ $(N, P, r, \lambda, D E, C E, \Phi)$, we define the package repository $R(d)$ as follows:

- the packages $P$ are defined as $\{(n, 1) \mid n \in N\}$, so we have a package for each node in the diagram, with a unique version number, 1

$-\forall$ node $n$ with sons $n_{1}, \ldots, n_{k}$, add dependencies as follows:

- if $\lambda(n)=$ or, add $n_{1}, \ldots, n_{k}$ as disjunctive dependencies for $n$

- if $\lambda(n)=$ and, add $n_{1}, \ldots, n_{k}$ as conjunctive dependencies for $n$

- if $\lambda(n)=x o r$, add $n_{1}, \ldots, n_{k}$ as disjunctive dependencies of $n$, and add $n_{1}, \ldots n_{i-1}, n_{i+1}, \ldots, n_{k}$ as conflicts for $\forall n_{i}$

$-\forall$ node $n$ with son $n^{\prime}$ and $\lambda(n)=$ opt, add $n^{\prime}$ as a recommend of $n$

- $\forall$ constraint c in $C E$ or $\Phi$, add the following dependencies:

- if c is $n \Rightarrow n^{\prime}$ or $n$ implies $n^{\prime}$, add $n^{\prime}$ as a conjunctive dependency of $n$

- if $c$ is $n \mid n^{\prime}$ or $n$ mutex $n^{\prime}$, add $n^{\prime}$ to the conflicts of $n$

- if $G T=$ Tree, $\forall n \rightarrow n^{\prime} \in D E$, add $n$ as a conjunctive dependency for $n^{\prime}$

- if $G T=D A G, \forall n \neq r$, add a dependency on $n_{1}|\cdots| n_{k}$ where $n_{1}, \ldots, n_{k}$ are all the parents of $n$ 

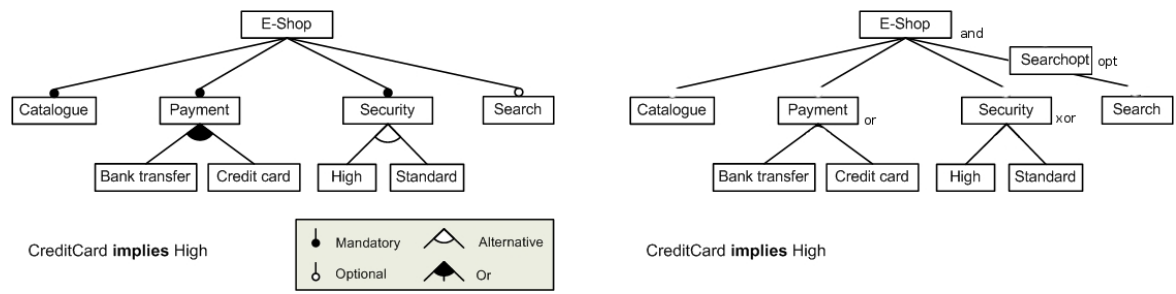

CreditCard implies High

Fig. 1. Sample E-Shop feature model: as FD (on the left) and FFD (on the right).

An even more compact, linear-space encoding for justifications and xor nodes can be given using virtual packages [7], exploiting the property that self-conflicts are ignored; it has been omitted here due to space constraints.

Example 1. A feature model using an edge-based semantics for an e-shop is shown in Figure 1 as FD (on the left) and FFD (on the right). Its encoding as package repository is reported below, where we drop Version: 1.

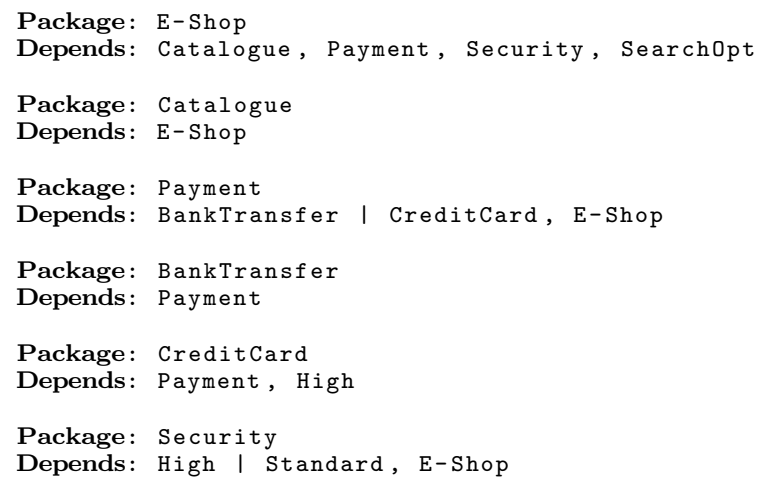

Notice how all kinds of metadata are used: conflicts encode mutual exclusion, recommends encode optional features, conjunctive depends encode and nodes and implications, disjunctive dependencies encode or nodes. It is now possible to establish the key property of the detailed encoding.

Theorem 1 (Soundness and completeness). A subset $M \subseteq N$ of the nodes of a $d \in F F D_{\text {core }}$ is a valid model of $d$ if and only if $m$ is a healthy installation for the package repository encoding $R(d)$.

Proof. The proof is by case analysis on the definition of a valid model, and the structure of the encoding. Details are omitted due to lack of space.

\section{Conclusions and future work}

We have established a direct mapping from a significant subset of Free Feature Diagrams to packages of FOSS distributions. This paves the way to reuse of theo- 
retical results as well as tools coming from FOSS research. Package management tools scale to tens of thousands packages and hundreds of thousands dependencies, and cope with evolving components. For instance, the edos.debian.net site provides quality metrics for FOSS distributions comprising more than 20'000 packages and 400'000 dependencies, daily, since 2006. Also, model construction with respect to user-defined optimizations is implemented by several tools, and competitions like www.mancoosi.org/misc-2010 are improving their efficiency.

We plan to extend the current encoding to all FFD constructs such as cardinality constraints and to validate the proposed approach by providing a full toolchain that attacks SPL problems using existing package management technology.

We hope that our work will contribute to establish a connection between SPLs and package management, for the joint benefit of both communities.

\section{References}

1. Abate, P., Boender, J., Di Cosmo, R., Zacchiroli, S.: Strong dependencies between software components. In: ESEM 2009. pp. 89-99. IEEE (2009)

2. Batory, D.: Feature models, grammars, and propositional formulas. In: SPLC 2005. LNCS, vol. 3714, pp. 7-20. Springer (2005)

3. Benavides, D., Martín-Arroyo, P.T., Cortés, A.R.: Automated reasoning on feature models. In: CAiSE. LNCS, vol. 3520, pp. 491-503. Springer (2005)

4. Berre, D.L., Rapicault, P.: Dependency management for the Eclipse ecosystem. In: IWOCE'09. ACM (2009)

5. Boender, J., Di Cosmo, R., Vouillon, J., Durak, B., Mancinelli, F.: Improving the quality of GNU/Linux distributions. In: COMPSAC. pp. 1240-1246. IEEE (2008)

6. de Jonge, M., Visser, J.: Grammars as feature diagrams. In: ICSR7 Workshop on Generative Programming. pp. 23-24 (2002)

7. Di Cosmo, R., Trezentos, P., Zacchiroli, S.: Package upgrades in FOSS distributions: Details and challenges. In: HotSWup'08. ACM (2008)

8. EDOS project, WP2 team: Report on formal management of software dependencies. Deliverable Work Package 2, Deliverable 2 (2006)

9. Janota, M.: Do sat solvers make good configurators? In: SPLC 2008, Second Volume (Workshops). pp. 191-195 (2008)

10. Janota, M., Kiniry, J.: Reasoning about feature models in higher-order logic. In: SPLC. pp. 13-22. IEEE Computer Society (2007)

11. Kang, K.C., Cohen, S.G., Hess, J.A., Novak, W.E., Peterson, A.S.: Feature-oriented domain analysis (FODA) feasibility study. Tech. rep., CMU (1990)

12. Le Berre, D., Parrain, A.: On SAT technologies for dependency management and beyond. In: ASPL'08 (2008)

13. Mancinelli, F., Boender, J., Di Cosmo, R., Vouillon, J., Durak, B., Leroy, X., Treinen, R.: Managing the complexity of large free and open source package-based software distributions. In: ASE 2006. pp. 199-208. IEEE (2006)

14. Schobbens, P.Y., Heymans, P., Trigaux, J.C.: Feature diagrams: A survey and a formal semantics. In: RE'06. pp. 136-145. IEEE (2006)

15. Treinen, R., Zacchiroli, S.: Solving package dependencies: from EDOS to Mancoosi. In: DebConf 8: proceedings of the 9th conference of the Debian project (2008)

16. Tucker, C., Shuffelton, D., Jhala, R., Lerner, S.: OPIUM: Optimal package install/uninstall manager. In: ICSE 2007. pp. 178-188 (2007) 